\title{
Projective Synchronization of Chaotic Systems Via Backstepping Design
}

\author{
${ }^{1}$ Anindita Tarai (Poria), ${ }^{2}$ Mohammad Ali Khan \\ ${ }^{1}$ Department of Mathematics, Aligunj R.R.B. High School Midnapore \\ (West), West Bengal, India \\ E-mail: mdmaths80@yahoo.com \\ ${ }^{2}$ Department of Mathematics, Garhbeta Ramsundar Vidyabhavan, Garhbeta, \\ Midnapore (West), West Bengal, India \\ E-mail: mdmaths@gmail.com
}

\begin{abstract}
Chaos synchronization of discrete dynamical systems are investigated. An algorithm is proposed for projective synchronization of chaotic 2D Duffing map and chaotic Tinkerbell map. The control law was derived from the Lyapunov stability theory. The numerical simulation results are presented to verify the effectiveness of the proposed algorithm
\end{abstract}

Keywords: Lyapunov function, Projective synchronization, Backstepping Design, Duffing map and Tinkerbell map.

\section{Introduction}

Adjacent chaotic trajectories governed by the same nonlinear systems may evolve into a state utterly uncorrelated, but in 1990 Pecora and Carrol [1] shown that it could be synchronized through a proper coupling. Since their seminal paper in 1990, chaos synchronization is an interesting research topic of great attention. Hayes et.al. (1993)[2] have studied to some potential applications in secure communication. Blasius et.al. [3] have observed complex dynamics and phase synchronization in spatially extended ecological systems in 1999 and system identification was investigated by Kocarev et.al. in 1996 [4]. Different forms of synchronization phenomena have been observed in a variety of chaotic systems, such as identical synchronization [1]. In 1996 Rosenblum et.al. [5] have studied phase synchronization of chaotic oscillators. A generalized synchronization for unidirectionally coupled dynamical systems was proposed by Rulkov et.al. [6] in 
1995, where two systems are called synchronized if a static functional relation exists between the states of the systems. The linear generalized chaos synchronization and predictability have investigated by S.Poria in 2007 [7]. Recently, in 2009 Khan et.al. [8] have studied generalized chaos synchronization of coupled Rossler system. A generalized anti-synchronization of different chaotic systems have investigated by Khan et.al. in 2012 [9]. In 2009 Tarai et.al. [10] have studied synchronization of bi-directionally coupled chaotic Chen's system with delay and they also studied generalized synchronization of linearly bidirectionally coupled unified chaotic system in 2009 [11].

Along with the development of control technology [12], the requirement for control precision gets higher and higher. In order to achieve the satisfied control performance, nonlinear control techniques are used. Backstepping is one of these nonlinear control techniques that has attracted a great deal of research interest in last few years. In 2003, Yongguang Y et. al. [13] have studied controlling uncertain Lu system using backstepping design. In 2001, adaptive synchronization of uncertain chaotic systems via backstepping design was investigated by Wang et. al [14]. Tan et.al. [15] applied backstepping design for synchronizing of chaotic systems. Chaos control of 4D chaotic systems using recursive backstepping nonlinear controller have investigated by Laoye et.al. in 2007 [16].

The key idea of the backstepping approach is to recursively design controllers for subsystems in the structure and "step back" the feedback signals towards the control input. This differs from the conventional feedback linearization in that it offers a more flexible way of dealing with uncertainties. Using the Lyapunov functions, the system can be kept into account and harmful nonlinearities can be cancelled or dominated by the control signal.

In 1998, J.M. Gonzalez-Miranda [17] derived a general condition for projective synchronization (PS) in arbitrary dimensional systems. In projective synchronization the drive and response vectors synchronize up to a scaling factor. PS is characterized by a scale factor that defines a proportional relation between the synchronized systems. PS results from the partial linearity of coupled chaotic systems, and become the unique feature of partially linear systems, studied by Rehacek et.al.[18] in 1999. The proportionality allows us to duplicate a chaotic system with different scales, while the topological characteristic of the two synchronized systems remain unchanged.

\section{Backstepping Design for Duffing Map}

We consider the drive Duffing Map as

$$
\begin{aligned}
x_{1}(k+1) & =x_{2}(k) \\
x_{2}(k+1) & =-b x_{1}(k)+a x_{2}(k)-x_{2}^{3}(k)
\end{aligned}
$$

and driven Duffing map as 


$$
\begin{aligned}
& y_{1}(k+1)=y_{2}(k) \\
& y_{2}(k+1)=-b y_{1}(k)+a y_{2}(k)-y_{2}^{3}(k)+u(k)
\end{aligned}
$$

Here $a$ and $b$ are the parameters of the Duffing map. Uncontrolled Duffing map displays chaos for $a=2.75$ and $b=0.2$. Now our aim is to find $u(k)$ such that the drive and response vectors become proportional, i.e., $\lim _{n \rightarrow \infty} \frac{y_{i}(n)}{x_{i}(n)}=\alpha$. Defining the errors as $e_{1}(k)=\alpha y_{1}(k)-x_{1}(k), e_{2}(k)=\alpha y_{2}(k)-x_{2}(k)$ we obtain the dynamical system for the error as

$$
\begin{aligned}
& e_{1}(k+1)=e_{2}(k) \\
& e_{2}(k+1)=-b e_{1}(k)+a e_{2}(k)-\alpha y_{2}^{3}(k)+x_{2}^{3}(k)+\alpha u(k)
\end{aligned}
$$

Now the systems (1) and (2) will synchronize if for suitable choice of $u(k)$ the error system stabilizes at the origin.

Following backstepping technique we assume $z_{1}(k)=e_{1}(k)$ and choose the Lyapunov function $V_{1}(k)=\left|e_{1}(k)\right|$. Then the variation of $V_{1}(k)$ with time is

$$
\begin{aligned}
& \Delta V_{1}(k)=V_{1}(k+1)-V_{1}(k) \\
& =\left|e_{1}(k+1)\right|-\left|e_{1}(k)\right| \\
& =\left|e_{2}(k)\right|-\left|z_{1}(k)\right| \\
& =\left|e_{2}(k)-z_{2}(k)+z_{2}(k)\right|-\left|z_{1}(k)\right| \\
& =\left|\beta(k)+z_{2}(k)\right|-\left|z_{1}(k)\right| \quad \text { introducting } z_{2}(k)=e_{2}(k)-\beta(k) \\
& =\left|c_{1} z_{1}(k)+z_{2}(k)\right|-\left|z_{1}(k)\right| \quad \text { choosing } \beta(k)=c_{1} z_{1}(k) \\
& \leq\left|z_{2}(k)\right|+\left(\left|c_{1}\right|-1\right)\left|z_{1}(k)\right|
\end{aligned}
$$

where $c_{1}$ is a design constant to be chosen later.

Now

$$
z_{1}(k+1)=e_{1}(k+1)=e_{2}(k)=z_{2}(k)+\beta(k)=c_{1} z_{1}(k)+z_{2}(k)
$$

Again,

$$
\begin{aligned}
z_{2}(k+1) & =e_{2}(k+1)-\beta(k+1) \\
& =-b e_{1}(k)+a e_{2}(k)-\alpha y_{2}^{3}(k)+x_{2}^{3}(k)+\alpha u(k)-c_{1}\left(c_{1} z_{1}(k)+z_{2}(k)\right)
\end{aligned}
$$

At this step we can determine the control law $u(k)$ as 


$$
u(k)=\left[c_{2} z_{2}(k)+b e_{1}(k)-a e_{2}(k)+\alpha y_{2}^{3}(k)-x_{2}^{3}(k)+c_{1}\left(c_{1} z_{1}(k)+z_{2}(k)\right)\right] / \alpha
$$

where $c_{2}$ is another design constant to be choosen later. Now with this $u(k)$ equation (7) becomes

$$
z_{2}(k+1)=c_{2} z_{2}(k)
$$

Choosing the second Lyapunov function as $V_{2}(k)=V_{1}(k)+d\left|z_{2}(k)\right|$, where $d$ is a positive constant, then the variation of $V_{2}(k)$ can be computed as

$$
\begin{aligned}
& \Delta V_{2}(k)=V_{2}(k+1)-V_{2}(k) \\
& =V_{1}(k+1)+d\left|z_{2}(k+1)\right|-V_{1}(k)-d\left|z_{2}(k)\right| \\
& =\Delta V_{1}(k)+d\left|z_{2}(k+1)\right|-d\left|z_{2}(k)\right| \\
& \leq\left|z_{2}(k)\right|+\left(\left|c_{1}\right|-1\right)\left|z_{1}(k)\right|+d\left|c_{2}\right|\left|z_{2}\right|-d\left|z_{2}(k)\right| \\
& =\left(\left|c_{1}\right|-1\right)\left|z_{1}(k)\right|+\left(d\left|c_{2}\right|-d+1\right)\left|z_{2}(k)\right|
\end{aligned}
$$

We choose $c_{1}$ and $c_{2}$ such that the right hand side of (9) become negative definite. Clearly the right hand side of (9) is negative definite if we choose $\left|c_{1}\right|<1$ and $\left|c_{2}\right|<\frac{d-1}{d}$ where $d>1$. We can choose the parameter $d$ to be large enough. Hence the close loop system (5) and (8)

$$
\left[\begin{array}{c}
z_{1}(k+1) \\
z_{2}(k+1)
\end{array}\right]=\left(\begin{array}{cc}
c_{1} & 1 \\
0 & c_{2}
\end{array}\right)\left[\begin{array}{c}
z_{1}(k) \\
z_{2}(k)
\end{array}\right]=A\left[\begin{array}{c}
z_{1}(k) \\
z_{2}(k)
\end{array}\right]
$$

is globally stable about the origin. Therefore system (1) is synchronized with system (2) up to a constant scaling factor $\alpha$. Notice that if we choose any one of the design constants $c_{1}, c_{2}$ to be zero, the dead-beat response will happen since $A^{2}=0$, which implies that the error state will be bought to zero in atmost 2 time steps. Therefore projective synchronization in finite time can be achieved. 


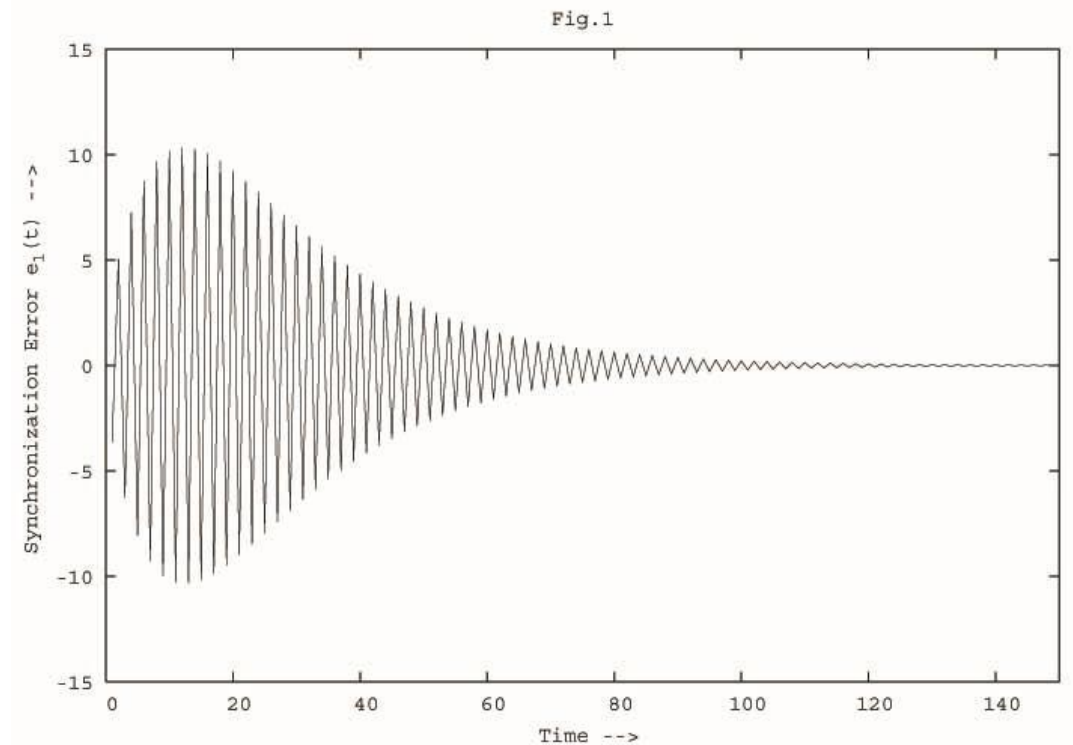

Figure 1: Time evolution of the synchronization error $\mathrm{e}_{1}(\mathrm{t})$ for $\mathrm{c}_{1}=-0.9, \mathrm{c}_{2}=-0.95$ are drawn for Duffing map.

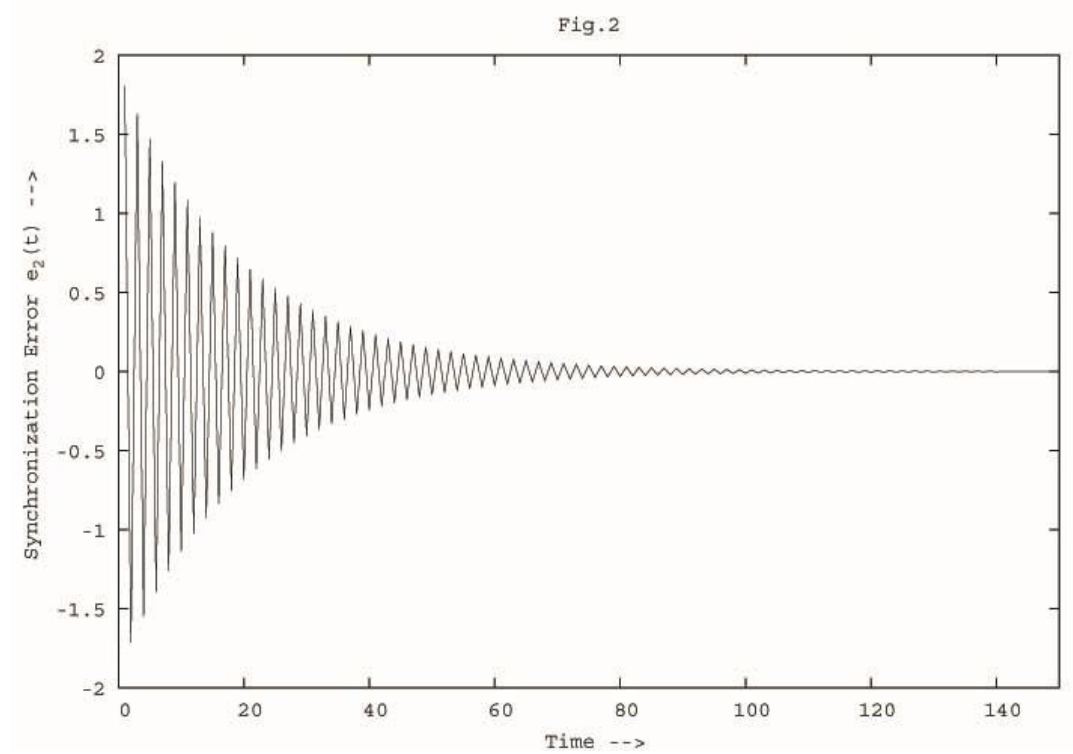

Figure 2: Time evolution of the synchronization error $\mathrm{e}_{2}(\mathrm{t})$ for $\mathrm{c}_{1}=-0.9, \mathrm{c}_{2}=-0.95$ are drawn for Duffing map.

\section{Backstepping Design for Tinkerbell Map}

We consider the driving Tinkerbell's map as

$$
x_{1}(k+1)=x_{1}^{2}(k)-x_{2}^{2}(k)+a x_{1}(k)+b x_{2}(k)
$$




$$
x_{2}(k+1)=2 x_{1}(k) x_{2}(k)+c x_{1}(k)+d x_{2}(k)
$$

and driven Tinkerbell's map as

$$
\begin{aligned}
& y_{1}(k+1)=y_{1}^{2}(k)-y_{2}^{2}(k)+a y_{1}(k)+b y_{2}(k) \\
& y_{2}(k+1)=2 y_{1}(k) y_{2}(k)+c y_{1}(k)+d y_{2}(k)+u(k)
\end{aligned}
$$

Here $a, b, c d$ are parameters of the map. Uncontrolled map displays chaos for $a=0.9, b=0.6013, c=2$ and $d=0.5$. Now our aim is to find an controller $u(k)$ such that the drive and response system synchronize projectively. Defining the errors as $e_{1}(k)=\alpha y_{1}(k)-x_{1}(k), e_{2}(k)=\alpha y_{2}(k)-x_{2}(k)$ we obtain the dynamical system for the error as

$$
\begin{aligned}
& e_{1}(k+1)=a e_{1}(k)+b e_{2}(k)+\alpha\left(y_{1}^{2}(k)-y_{2}^{2}(k)\right)-\left(x_{1}^{2}(k)-x_{2}^{2}(k)\right) \\
& e_{2}(k+1)=c e_{1}(k)+d_{2}(k)+2 \alpha\left(y_{1}(k) y_{2}(k)-x_{1}(k) x_{2}(k)\right)+\alpha u(k)
\end{aligned}
$$

For synchronization of (11) and (12) the error system should be stabilized at origin. According to backstepping design we assume $z_{1}(k)=e_{1}(k)$. Choosing the Lyapunov function as $V_{1}(k)=\left|e_{1}(k)\right|$, we get the time variation of $V_{1}(k)$ as

$$
\begin{aligned}
\Delta V_{1}(k) & =\left|e_{1}(k+1)\right|-\left|e_{1}(k)\right| \\
& =\mid \alpha\left(y_{1}^{2}(k)-y_{2}^{2}(k)\right)-\left(x_{1}^{2}(k)-x_{2}^{2}(k)+a e_{1}(k)+b e_{2}(k)|-| z_{1}(k) \mid\right.
\end{aligned}
$$

We choose

$$
\beta(k)=c_{1} z_{1}(k)-\alpha\left(y_{1}^{2}(k)-y_{2}^{2}(k)\right)-\left(x_{1}^{2}(k)-x_{2}^{2}(k)=c_{1} z_{1}(k)+\phi(k)(s a y)\right.
$$

here $c_{1}$ is a constant to be chosen later and introduce the variable $z_{2}(k)=e_{2}(k)-\beta(k)$. Now,

$$
\begin{aligned}
& \Delta V_{1}(k)=\left|\alpha\left(y_{1}^{2}(k)-y_{2}^{2}(k)\right)-\left(x_{1}^{2}(k)-x_{2}^{2}(k)\right)+a z_{1}(k)+b e_{2}(k)+z_{2}(k)-z_{2}(k)\right|-\left|z_{1}(k)\right| \\
& \leq\left|\alpha\left(y_{1}^{2}(k)-y_{2}^{2}(k)\right)-\left(x_{1}^{2}(k)-x_{2}^{2}(k)\right)+b e_{2}(k)+z_{2}(k)-z_{2}(k)\right|+(a-1)\left|z_{1}(k)\right| \\
& =\left|\beta(k)+z_{2}(k)+\alpha\left(y_{1}^{2}(k)-y_{2}^{2}(k)\right)-\left(x_{1}^{2}(k)-x_{2}^{2}(k)\right)\right|+(a-1)\left|z_{1}(k)\right| \\
& =\left|c_{1} z_{1}(k)+z_{2}(k)\right|+(a-1)\left|z_{1}(k)\right| \\
& \leq\left|z_{2}(k)\right|+\left(\left|c_{1}\right|+a-1\right)\left|z_{1}(k)\right|
\end{aligned}
$$

Therefore 


$$
\begin{aligned}
z_{1}(k+1)=e_{1}(k+1)=\alpha\left(y_{1}^{2}(k)-y_{2}^{2}(k)\right)-\left(x_{1}^{2}(k)-x_{2}^{2}(k)\right)+a e_{1}(k)+b e_{2}(k) \\
=\alpha\left(y_{1}^{2}(k)-y_{2}^{2}(k)\right)-\left(x_{1}^{2}(k)-x_{2}^{2}(k)\right)+a z_{1}(k)+z_{2}(k)+\beta(k) \\
=c_{1} z_{1}(k)+a z_{1}(k)+z_{2}(k) \\
=\left(c_{1}+a\right) z_{1}(k)+z_{2}(k)
\end{aligned}
$$

Now we have

$$
\begin{aligned}
z_{2}(k+1) & =b e_{2}(k+1)-\beta(k+1) \\
& =b\left[2 \alpha\left(y_{1}(k) y_{2}(k)-x_{1}(k) x_{2}(k)\right)+c e_{1}(k)+d e_{2}(k)+\alpha u(k)\right] \\
& -c_{1}\left[\left(c_{1}+a\right) z_{1}(k)+z_{2}(k)\right]-\phi(k+1)
\end{aligned}
$$

At this step we choose the control lae $u(k)$ as

$$
\mathrm{u}(\mathrm{k})=\left[\begin{array}{l}
\mathrm{c}_{2} \mathrm{z}_{2}(\mathrm{k})-2 \mathrm{~b} \alpha\left(\mathrm{y}_{1}(\mathrm{k}) \mathrm{y}_{2}(\mathrm{k})-\mathrm{x}_{1}(\mathrm{k}) \mathrm{x}_{2}(\mathrm{k})\right) \\
-\mathrm{cbe}_{1}(\mathrm{k})-\mathrm{bde}_{2}(\mathrm{k})+\mathrm{c}_{1}\left\{\left(\mathrm{c}_{1}+\mathrm{a}\right) \mathrm{z}_{1}(\mathrm{k})+\mathrm{z}_{2}(\mathrm{k})\right\}
\end{array}\right]+\phi(k+1) / b \alpha
$$

With this choice we get

$$
z_{2}(k+1)=c_{2} z_{2}(k)
$$

Let us take second Lyapunov function as $V_{2}(k)=V_{1}(k)+d\left|z_{2}(k)\right|$ where $d$ is a positive constant. Then the time variation of $V_{2}(k)$ is given by

$$
\begin{aligned}
& \Delta V_{2}(k)=V_{2}(k+1)-V_{2}(k) \\
& =\Delta V_{1}(k)+d\left|z_{2}(k+1)\right|+d\left|z_{2}(k)\right| \\
& \leq\left|z_{2}(k)\right|+\left(\left|c_{1}\right|+a-1\right)\left|z_{1}(k)\right|+d\left|c_{2}\right|\left|z_{2}(k)\right|-d\left|z_{2}(k)\right| \\
& =\left(\left|c_{1}\right|+a-1\right)\left|z_{1}(k)\right|+\left(d\left|c_{2}\right|-d+1\right)\left|z_{2}(k)\right|
\end{aligned}
$$

In order to make the right hand side of (21) negative definite we have to choose $a-1<c_{1}<1-a,\left|c_{2}\right|<\frac{d-1}{d}$ where $d>1$. Now the close-loop system (17) and (20) become

$$
\left[\begin{array}{c}
z_{1}(k+1) \\
z_{2}(k+1)
\end{array}\right]=\left(\begin{array}{cc}
c_{1}+a & 1 \\
0 & c_{2}
\end{array}\right)\left[\begin{array}{c}
z_{1}(k) \\
z_{2}(k)
\end{array}\right]=A\left[\begin{array}{c}
z_{1}(k) \\
z_{2}(k)
\end{array}\right]
$$

is globally stable about the origin. Therefore system (1) is synchronized with system (2) up to a constant scaling factor $\alpha$. Notice that if we choose any one of the design constants $c_{1}=-a$, or $c_{2}$ to be zero, the dead-beat response will happen since $A^{2}=0$, which implies that the error state will be bought to zero in atmost 2 time steps. Therefore projective synchronization in finite time can be achieved. 


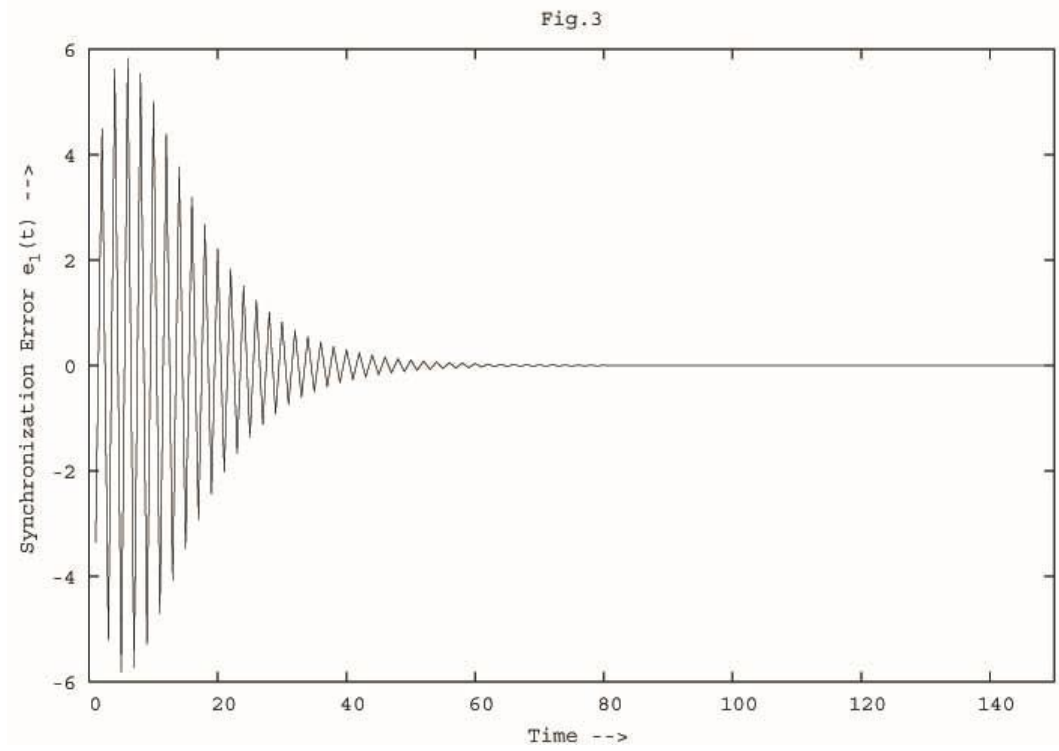

Figure 3: Time evolution of the synchronization error $\mathrm{e}_{1}(\mathrm{t})$ for $\mathrm{c}_{1}=-1.7, \mathrm{c}_{2}=-0.9$ are drawn for Tinkerbell map.

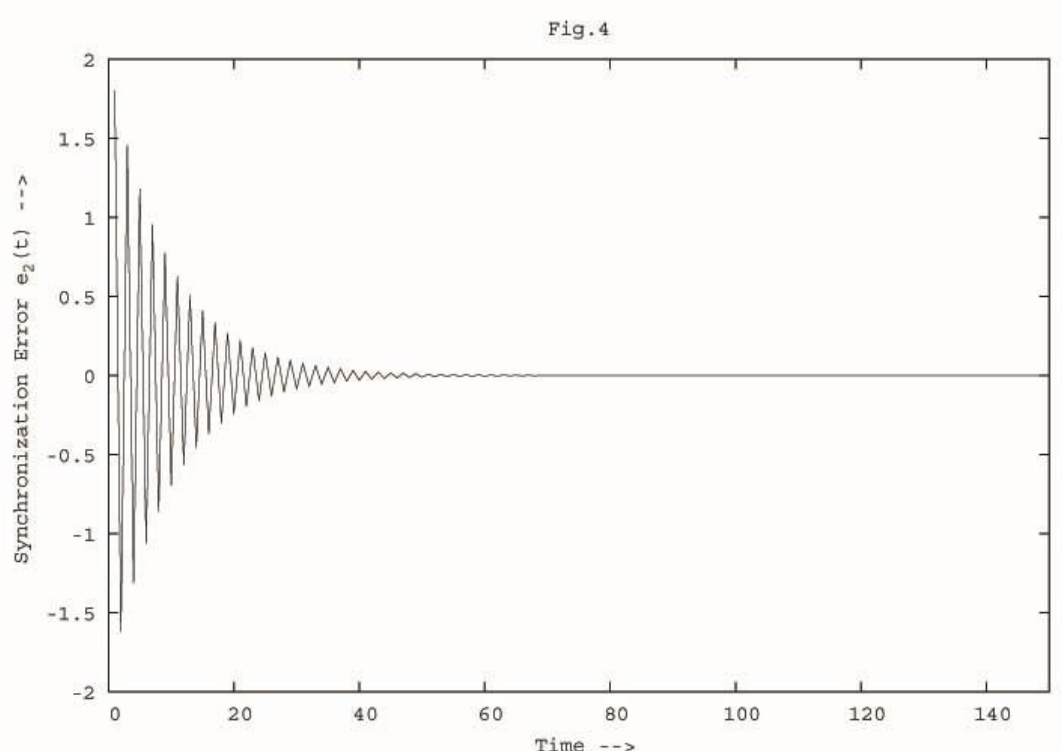

Figure 4: Time evolution of the synchronization error $\mathrm{e}_{2}(\mathrm{t})$ for $\mathrm{c}_{1}=-1.7, \mathrm{c}_{2}=-0.9$ are drawn for Tinkerbell map.

\section{Results and Discussions}

We have successfully applied the backstepping method for projective synchronization of Duffing map and Tinkerbell map. The numerical simulation 
results for the case of Duffing map are shown in Fig.1 and Fig.2 taking the value of $c_{1}=-0.9$ and $c_{2}=-0.95$. In both the cases the error goes to zero through oscillation. We assume the synchronization errors was initially $e_{1}(t)=1.9$ and $e_{2}(t)=-1.9$. Simulation results for the case of Tinkerbell map are shown in Fig.4 and Fig.5 taking the values of $c_{1}=-1.7$ and $c_{2}=-0.9$. Here also the error goes to zero in a oscillatory way. The numerical simulation results indicate that this approach works very well.

\section{References}

[1] L.M. Pecora and T.L. Carroll, Synchronization in chaotic systems, Phys. Rev. Lett, 64 (1990) 821-824.

[2] S.Hayes, C.Grebogi and E.Ott, Communicating with chaos, Phys. Rev. Lett. 70(1993) 3031-3034.

[3] B. Blasius, A.Huppert, L.Stone, Complex dynamics and phase synchronization in spatially extended ecological systems, Nature, 399 (1999) 354-359.

[4] U. Parlitz, L.Junge and L.Kocarev, Synchronization-based parameter estimation from time series, Phys. Rev. E, 54 (1996) 6253-6259.

[5] M. Rosenblum, A.Pikovosky and J.Kurths, Phase synchronization of chaotic oscillators, Phys. Rev. Lett,76 (1996) 1804-1807.

[6] N. F. Rulkov, M.M.Suschik and L.S.Tsimring, Generalized synchronization of chaos in directionally coupled chaotic systems, Phys. Rev.E. 51 (1995) 980-994.

[7] S. Poria, The linear generalized chaos synchronization and predictability, Int.J.of Applied Mechanics and Engineering, 12(2007) 879-885.

[8] M. A. Khan and A.K.Mandal, Generalized chaos synchronization of coupled Rossler systems, Bull.Cal.Math.Soc, 101(2009) 197-204.

[9] M. A. Khan, S.N.Pal and S.Poria, Generalized anti-synchronization of different chaotic systems, Int. J. of Applied Mechanics and Engineering, 17(2012) 83-89.

[10] A. Tarai(Poria), S. Poria and P. Chatterjee, Synchronization of bidirectionally coupled chaotic Chen's system with delay, Chaos Solitons and Fractals, 41 (2009) 643-647.

[11] A. Tarai, S.Poria and P. Chatterjee, Synchronization of generalized linearly bidirectionally coupled unified system, Chaos Solitons and Fractals. 40 (2009) 885-892.

[12] M. A. Khan, A. K. Mondal and S. Poria, Three control strategies for unified chaotic system, Int. J. of Applied Mechanics and Engineering, 16 (2011) 597-608.

[13] Yongguang Y, Suochun Z., Controlling uncertain Lu system using backstepping design, Chaos, Solitons \& Fractals, 15 (2003) 897-902. 
[14] C.Wang and S.S. Ge, Adaptive synchronization of uncertain chaotic systems via backstepping design, Chaos,Solitons \& Fractals, 12 (2001) 1199-1206.

[15] X.Tan, J.Zhang and Y.Yang, Synchronizing chaotic systems using backstepping design, Chaos, Solitons \& Fractals 16 (2003) 37-45.

[16] J. A. Laoye, U. E. Vincent and S. O. Kareem, Chaos control of 4D chaotic systems using recursive backstepping nonlinear controller, Chaos,Solitons \& Fractals 32(2007) 1823-1829.

[17] J. M. Gonzalez-Miranda, Amplification and displacement of chaotic attractors by means of unidirectional chaotic driving, Phys. Rev. E, 57(1998) 7321-7324.

[18] R. Mainieri and J. Rehacek, Projective synchronization in three-demensional chaotic system, Phys. Rev. Lett., 82 (1999) 3042-3045. 